\title{
Génération d'UV-X mous par décharge électrique
}

\author{
J. M. Pouvesle et D. Pigache*
}

GREMI, Université d'Orléans, CNRS, UFR-SFA, BP. 6759, 45067 Orléans Cedex 2, France * Office National d'Études et de Recherches Aérospatiales, BP. 72, 92322 Chatillon Cedex, France

Les sources incohérentes de photons UV-X mous connaissent un regain d'intérêt dans grand nombre de domaines. Les décharges électriques, grâce à leur grande flexibilité (durée d'impulsion, énergies déposées, gamme de pression d'utilisation, tensions appliquées, géométries) restent l'outil privilégié pour la production de photons même d'énergie élevée. A l'heure actuelle, un effort considérable est fourni dans les laboratoires pour l'adaptation et l'optimisation de techniques classiques à la génération de photons, de même que de nouveaux types d'excitation sont mis en oeuvre. Il serait vain d'en dresser un panorama complet. Nous nous limiterons à quelques cas particuliers que nous aborderons par ordre de pression décroissante.

- Décharge silencieuse et décharge contrôlée par diélectrique [1]-[5]

- Décharge micro-onde dans les mélanges excimères [6]-[10]

- Décharge axiale dans les jets supersoniques [11]

- Décharge à faisceau d'électrons [12]-[14]

- Décharge du type pseudo-spark [15]-[21]

Les deux premières, connues depuis longtemps et utilisées pour diverses applications font l'objet de nouveaux travaux en tant que source UV et VUV incohérentes de très bon rendement. Les deux suivantes sont à retenir comme sources VUV et la dernière comme source d'X mous quasi ponctuelle et impulsionnelle. 


\section{Références}

[1] B. Gellert and U. Kogelschatz, Appl. Phys. B. 52, 14 (1991)

[2] B. Eliasson and U. Kogelschatz, IEEE Trans. Plasma Science 19, 309 (1991)

[3] K. Stockwald, V. Schorp, M. Neiger and C. Beneking, XX $X^{c}$ ICPIG Proceedings, 4, 953 (1991)

[4] C. Cachoncinlle, J.M. Pouvesle, F. Davanloo, J.J.Cogan and C.B. Collins, Opt. Comm. 79, 41 (1990)

[5] C. Cachoncinlle, J.M. Pouvesle, G. Durand and F. Spiegelmann, J. Chem. Phys. (parution 15 avril 1992)

[6] I. Nakamura, F. Kannari and M. Obara, Appl. Phys. Lett. 57, 2057 (1990)

[7] T. Hatakeyama, F. Kannari and M. Obara, Appl. Phys. Lett 59, 387 (1991)

[8] K. Yoshizawa, M. Taki, K. Tachibana and S. Moriyama, Appl. Phys. Lett. 59, 1678 (1991)

[9] S.B. Hassal and E.A. Ballik, J. Appl. Phys. 70, 1042 (1991)

[10] S.B Hassal and E.A. Ballik, Can. J. Phys. $\underline{69}, 699$ (1991)

[11] P. Dubé, T. Efthimiopoulos, M.J. Kiik and B.P. Stoicheff, Optics Letters 16, 1887 (1991)

[12] J.J. Rocca et al., J. Appl. Phys. $\underline{56}, 790$ (1984)

[13] A.M. Pointer et al., J. Appl. Phys. 65, 4632 (1989)

[14] Z. Yu et al., Appl. Phys. Lett. 57, 1873 (1990)

[15] J. Christiansen and Ch. Shultheiss, Z. Physik A. 290, 35 (1979)

[16] K. Franck et al., IEEE Trans. on Plasma Science 16,317 (1988) - Article de revue

[17] J.P.Boeuf and L.C. Pitchford, IEEE Trans. on Plasma Science 19, 286 (1991)

[18] P. Bickel et al., Proceedings 19th Power Modulator Symposium, San Diego (1990) p. 232

[19] C. Braun et al., IEEE Trans. on Electron Devices 35, 559 (1988)

[20] G. Jung et al., Proceedings Gas Discharges Conf. Venise (1988) p. 681

[21] C. Schultheiss, Communication privée 1992 